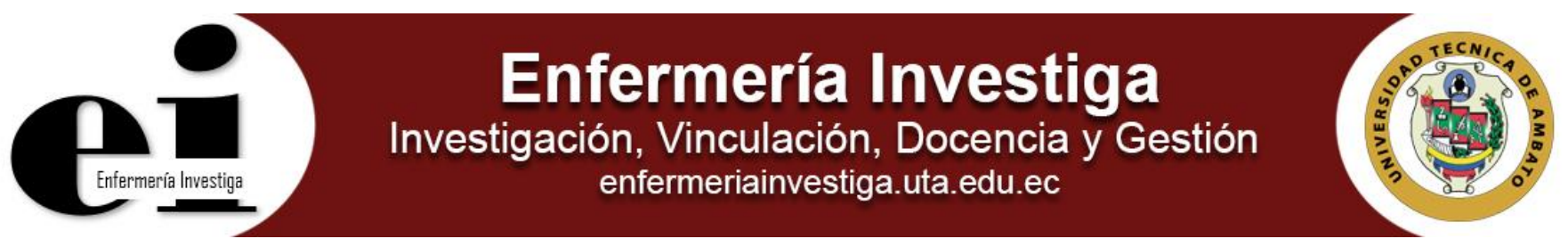

DOI: http://dx.doi.org/10.29033/ei.v3n1.2018.09

Presentación de casos

\title{
Hemangioendotelioma hepático del lactante
}

Infant hepatic Hemangioendothelioma

\author{
${ }^{1}$ Hospital Carlos Andrade Marín - Departamento de Radiología e Imagen - Quito - Ecuador \\ ${ }^{2}$ Hospital Carlos Andrade Marín - Departamento de Medicina - Quito - Ecuador \\ ${ }^{3}$ Hospital Carlos Andrade Marín - Departamento de Patología Clínica - Quito - Ecuador
}

Juan Carlos Jara Santamaría ${ }^{1}$, Esteban Israel Parreño Ortega², Johanna Carolina Prado Parreño ${ }^{3}$

Jara JC, Parreño El, Prado JC. Hemangioendotelioma hepático del lactante. Enferm Inv. 2017; 3(1):44-48.

2477-9172 / 2550-6692 Derechos Reservados @ 2017 Universidad Técnica de Ambato, Carrera de Enfermería. Este es un artículo de acceso abierto distribuido bajo los términos de la Licencia Creative Commons, que permite uso ilimitado, distribución y reproducción en cualquier medio, siempre que la obra original es debidamente citada.

\section{Historia:}

Recibido: 27 octubre 2017

Revisado: 11 enero 2018

Aceptado: 28 febrero 2018

Palabras Claves:

Hemangioendotelioma;

neoplasias; hígado; infante;

radiología

Keywords:

Hemangioendothelioma; neoplasms; liver; infant; radiology

\section{Resumen}

Introducción: El hemangioendotelioma hepático infantil es una afectación poco común que se caracteriza por el desarrollo de una o varias neoplasias de origen vascular en el hígado. Dichas neoplasias generalmente son benignas, aunque tienen un potencial intermedio de transformarse en tumores malignos.

Objetivo: Dar a conocer el caso de una patología de presentación inusual, enfatizar su importancia dentro de los diagnósticos diferenciales en estudios radiológicos con tomografía computarizada y ecografía abdominal.

Descripción del caso: En el siguiente caso se presenta a un paciente lactante de dos meses de edad, nacido a las 38 semanas de gestación sin ninguna complicación aparente. Tras presentarse a la consulta debido al desarrollo de síntomas propios de la enfermedad, y tras realizar estudios de imagen, tomografía computarizada multicorte corporal y ecografía abdominal, se determinó que el paciente padece de múltiples hemangioendoteliomas hepáticos, sometido a terapia farmacológica y respiratoria además tratamiento intervencionista como embolización, sin tener mejoría, complicándose su cuadro clínico con insuficiencia cardiaca rara en esta patología y progresa hasta su fallecimiento.

Conclusiones: El diagnóstico de Hemangioendotelioma Hepático del lactante se puede hacer sin la exigencia de una confirmación histológica cuando los hallazgos clínicos, analíticos y de imagen son muy sugestivos. Es raro el curso de esta enfermedad con Insuficiencia cardiaca y fallecimiento del lactante.

\section{Abstract}

Introduction:

Infantile

hepatic

hemangioendothelioma is an uncommon condition characterized by the development of one or several neoplasms of vascular origin in the liver. These neoplasms are usually benign, although they have an intermediate potential of transforming into malignant tumors.

Objetive: To make known the case of an unusual presentation pathology, to emphasize its importance within the differential diagnoses in radiological studies with computed tomography and abdominal ultrasound.

Case description: In the following case, a twomonth-old infant was presented, born at 38 weeks of gestation without any apparent complication. After presenting to the consultation due to the development of symptoms typical of the disease, and after performing imaging studies, multislice computerized tomography (MCT) and abdominal ultrasound, it was determined that the patient suffers from multiple hepatic hemangioendotheliomas, undergoing pharmacological and respiratory therapy besides, interventionist treatment as embolization, without improvement, complicating his clinical picture with rare heart failure in this pathology and progressing until his death.

Conclusions: The diagnosis of Hepatic Hemangioendothelioma of the infant can be made without the requirement of histological confirmation when the clinical, analytical and imaging findings are very suggestive. The course of this disease with cardiac insufficiency and infant death is rare. 


\section{Introducción}

Las lesiones vasculares hepáticas en niños son raras y su diagnóstico diferencial las clasifican en lesiones benignas, las cuales incluyen linfagioma, angiomiolipoma, malformación arteriovenosa, hemangioendotelioma y malignas, tales como el hepatoblastoma, angiosarcoma, hamartoma mesenquimatoso y metástasis del neuroblastoma. ${ }^{1}$

El término "hemangioendotelioma" fue acuñado en 1908 para referirse a tumores que se localizaban en el endotelio de los vasos sanguíneos. Se conoce que es potencialmente maligno, especialmente cuando este tumor se localiza en regiones paratraqueales, laringe, mediastino, corazón e hígado, siendo este último órgano más frecuente por su ubicación extracutánea, en donde se ven comúnmente comprometidos los dos lóbulos hepáticos.²

Los hemangiomas infantiles aparecen generalmente en los primeros meses de vida, por lo general antes de los dos meses de vida extrauterina. ${ }^{3,4}$

En la infancia, los hemangiomas son los tumores más comunes, antes de los seis meses de edad, en el $90 \%$ de los casos son de origen benigno y como consecuencia de alto flujo sanguíneo se presenta la dilatación de los vasos. Las fases evolutivas de estos tumores son:

1. Proliferativa: crecen rápidamente las lesiones.

2. Estacionaria: no se identifica cambios clínicos.

3. Involutiva: se la aprecia en el segundo año de vida, el $90 \%$ de los casos se resuelven a los 10 años, el $75 \%$ hacia los 7 años y el $50 \%$ desaparecen hasta el quinto año. ${ }^{1}$

Generalmente, el 80 - 90\% de los casos de hemangioendotelioma suelen mostrar sintomatología a partir de los dos meses de vida. Hay casos en los que la sintomatología de la enfermedad puede aparecer a partir de 12 a 18 meses y existen también casos en los que la sintomatología es rápida y fatal. ${ }^{5}$ De igual forma, la afectación en el hígado es multifocal, pero existen casos de tumores solitarios que dificultan el diagnóstico diferencial con una neoplasia hepática maligna, ya que estos son de gran tamaño o masas multifocales en un lactante o niño pequeño, y se incluye el hepatoblastoma, infiltración linfomatosa y metástasis de neuroblastoma, permitiendo diferenciar entre un solitario y multifocal. ${ }^{6}$

Para el diagnóstico, es fundamental la historia clínica y estudios de imagen, tales como la tomografía computarizada multicorte (TCM), ultrasonografía abdominal y la imagen por resonancia magnética (IRM). Adicionalmente es de ayuda la gammagrafía con eritrocitos o plaquetas radiomarcadas. ${ }^{1}$

Existen varias opciones terapéuticas, pero principalmente existen dos fases. Primeramente, cuando el paciente presenta descompensación cardíaca se debe tratar con diuréticos y digitálicos, luego se plantea disminuir el flujo sanguíneo del hemangioma mediante el uso de corticoides e interferón alfa y procedimientos quirúrgicos como embolización, ligadura de la arteria hepática, trasplante hepático y resección quirúrgica que es de primera elección para casos de tumores solitarios. ${ }^{6}$

\section{Descripción del caso}

Se trata de un lactante de dos meses de edad, que presentó al nacimiento lesiones rojizas compatibles con hemangiomas en región occipital, parietal y frontal de la cara (labios, nariz).

La madre refiere que el 30 de enero del 2017 acudió al Hospital Padre Carolo pues quince días antes notó un hemangioma situado en la nariz aumento de tamaño, el que sangro y le dificultó respirar, a la valoración en esa casa de salud, le realizaron radiografía simple de tórax y evidenciaron cardiomegalia. Se solicitó valoración por cardiología pediátrica en Hospital Metropolitano (Hospital privado de tercer nivel) (30/02/2017), se indicó ecografía cardiaca, en donde se evidenció insuficiencia pulmonar moderada, tricúspide severa, mitral leve, dilatación de cámaras derechas e izquierdas, foramen oval permeable derecha e izquierda y conducto arterioso anatómico de $0.3 \mathrm{~cm} \times 0.2 \mathrm{~cm} \times$ $0.3 \mathrm{~cm}$.

En el examen físico el infante se presentó ictérico, cianótico, adinámico, con una marcada distensión abdominal en cuadrante superior derecho, succión pobre, múltiples angiomas cutáneos y soplo sistólico grado IV/VI en el borde esternal izquierdo. A la palpación abdominal se evidenciaron varias masas pequeñas, no móviles, en cuadrante superior derecho, por lo cual se decidió realizar exámenes complementarios. En la biometría hemática se evidenció una moderada trombocitopenia, con hemoglobina y hematocrito bajo.

Sin obtener mejoría del tratamiento realizado acudió al Hospital Carlos Andrade Marín, donde fue valorado por parte servicio de pediatría (15/02/2017) para manejo integral y se solicitó ecografía de abdomen en búsqueda de hemangiomas hepáticos como causa de insuficiencia cardiaca, en el cual se evidenciaron múltiples imágenes hipoecogénicas dispersas, redondeadas, con vascularidad central y periférica al doppler color, la mayor de $17 \times 14 \mathrm{~mm}$ en segmento hepático VII, con lo cual se sospechó de lesiones tipo hemangioma hepático multifocal.

En el servicio de pediatría fue revalorado por cirugía cardiotorácica y otros servicios como hematología, cirugía vascular y gastroenterología, solicitando la realización de una TCM, para correlacionar los hallazgos de la ecografía (17/02/2017). Se informó cardiomegalia y atelectasias pasivas en pulmón, además hígado incrementado de tamaño 
de densidad heterogénea. En la fase arterial se apreciaron múltiples nódulos con realce periférico, las lesiones más pequeñas realzaron por completo distribuidas en todo el parénquima hepático, con predominio de localización periférica. Como hallazgo casual se reportó la presencia hernia inguinal indirecta derecha.

Se realizó angiografía hepatomesentérica y embolización de arteria hepática (21/03/2017), donde se evidenció la presencia de múltiples lesiones focales hipervasculares sin evidencia de shunts arteriovenosos y blush capilar de morfología redondeada o circunferencial de varios tamaños (hasta aproximadamente $2 \mathrm{~cm}$ ), distribuidos aleatoriamente en todo el parénquima hepático.

Cabe mencionar que el servicio de cardiología intervencionista realizó cateterismo donde se realizó angioplastia con colocación de balón en foramen oval, pero falleció por insuficiencia cardiaca congestiva secundaria al hemangioendotelioma hepático.

El caso que se reporta fue diagnosticado gracias a la realización de un estudio en un ecógrafo Samsung RS-80 y una TMC en un Somaton Definition Dual Source de 254 cortes, en el Hospital Carlos Andrade Marín de la ciudad de Quito, adquiriéndose imágenes de eco-tomografía y de tomografía con y sin contraste; además no fue comprobado con histopatología ya que estas lesiones son típicas y el diagnóstico casi siempre se realiza por tomografía contrastada.

Figura 1. Niñograma donde se evidencia cardiomegalia y hepatomegalia.

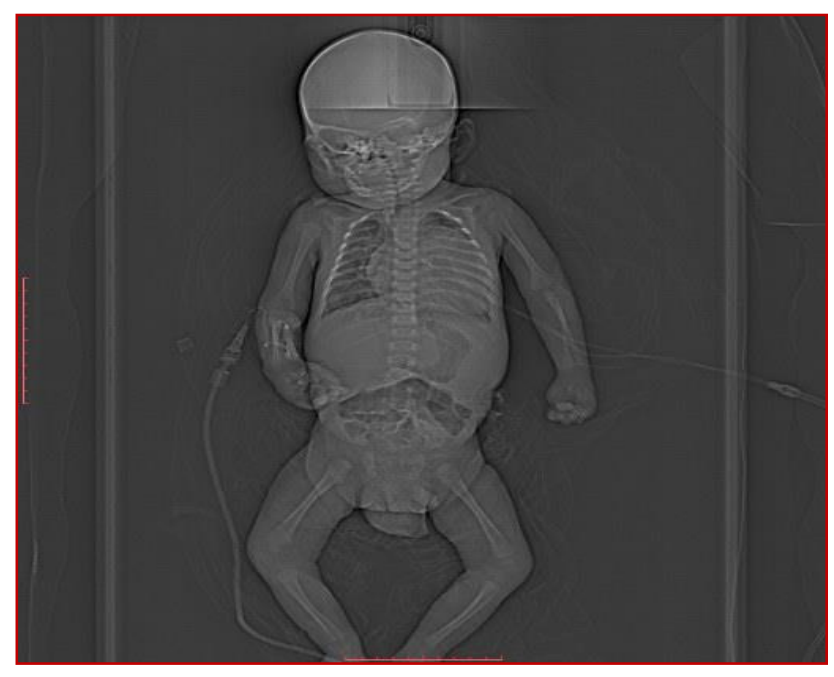

Al realizar una ecosonografía de abdomen donde se confirmó la hepatomegalia con ambos lóbulos comprometidos y se observaron múltiples imágenes redondeadas hipoecoicas (figura 2) y altamente vascularizadas al doppler color (figura 3).

Figura 2. Ecografía abdominal con hepatomegalia y múltiples imágenes redondeadas hipoecoicas.

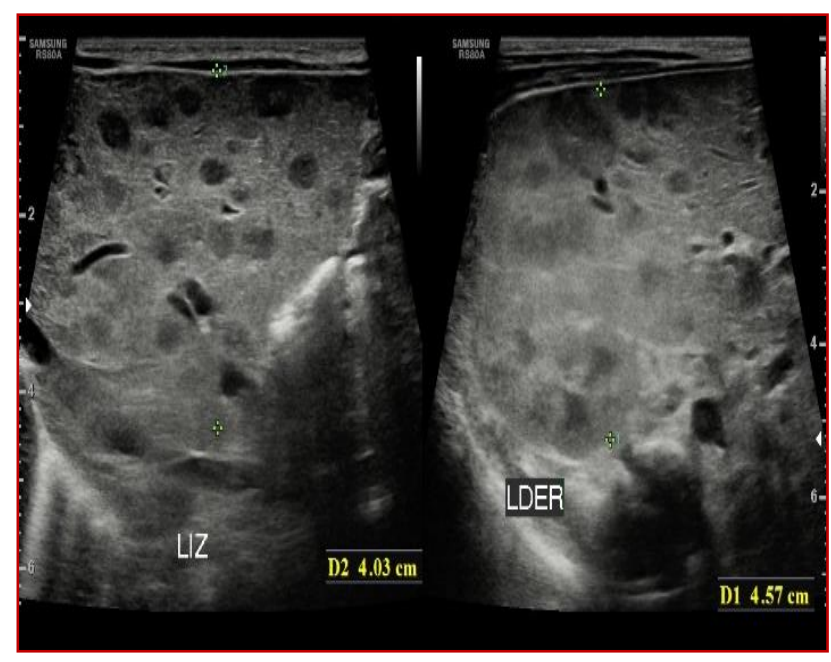


Figura 3. Ecografía abdominal donde se aprecia alta vascularización de hemangioendoteliomas, observada con técnica doppler color.

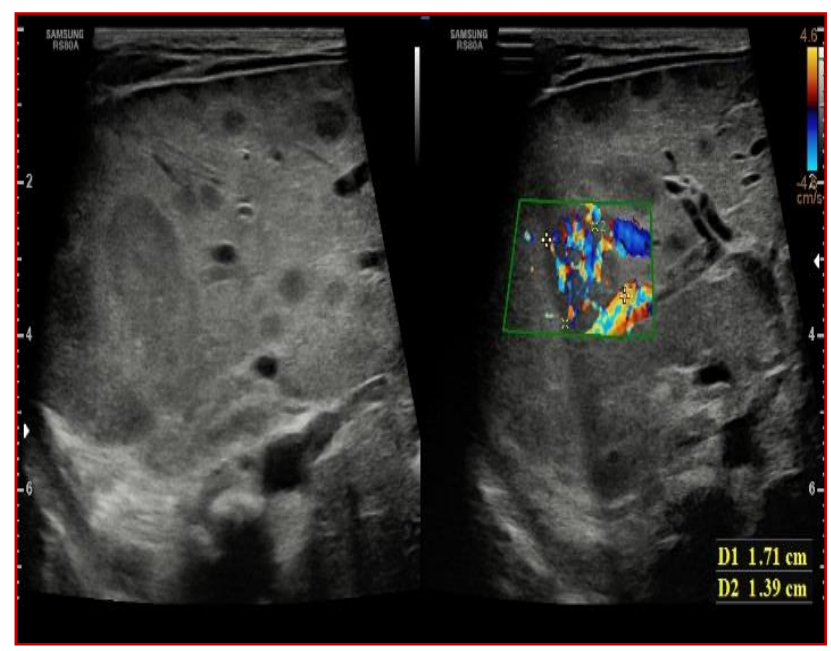

Se complementaron los estudios con la realización de tomografía.

Figura 4. Tomografía Computarizada Contrastada donde se evidencia cardiomegalia, atelectasias basales y hepatomegalia con realce periférico de los nódulos.

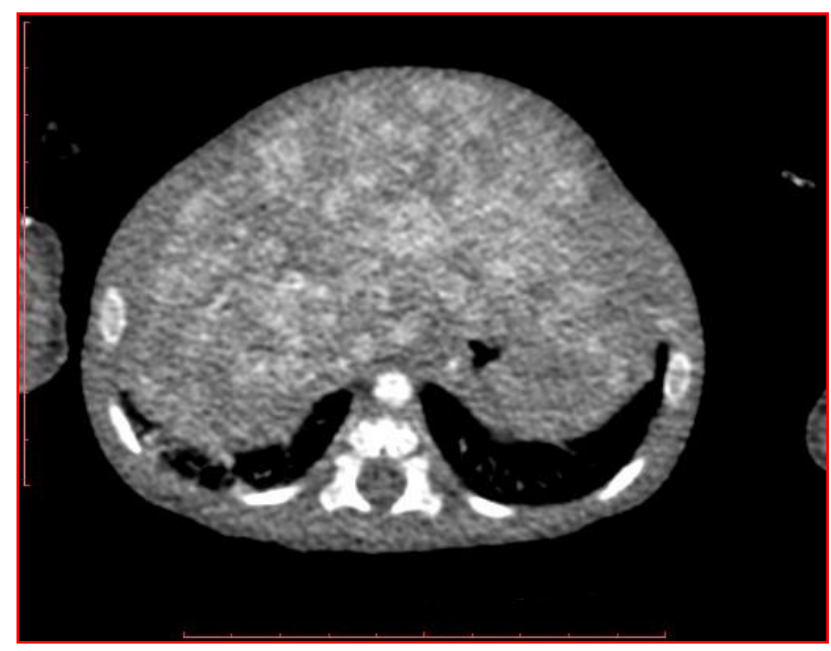

Después de ver estas imágenes tan típicas, se llegó al diagnóstico de hemangioendotelioma hepático infantil o del lactante $(\mathrm{HHI}$ ). El tratamiento a seguir fue sintomático con un monitoreo continuo para verificar si existía involución, en caso contrario. Entre las posibles complicaciones estaba la insuficiencia cardíaca.

\section{Discusión}

Los hemangiomas hepáticos son los tumores de hígado más comunes en la infancia, con mayor frecuencia e incidencia en menores de dos años, concretamente en la edad de lactancia y comúnmente en mujeres. Este tipo de tumores tienen una forma de presentación muy variable, se han descrito dos tipos de $\mathrm{HHI}$ : el tipo 1 donde el tumor tiene un aspecto cavernoso con dilatación de canales vasculares revestidos de células endoteliales que son citológicamente benignas, y el tipo 2 donde se aprecian canales vasculares irregulares con células endoteliales pleomórficas. Su diagnóstico se da mayormente de forma incidental debido a la forma de su presentación. ${ }^{7}$

Los hemangiomas hepáticos pueden aparecer como lesiones únicas o múltiples que abarcan ambos lóbulos, pero de afectación más frecuente en el lóbulo hepático izquierdo, en el caso que se presenta se evidencia un compromiso de ambos lóbulos. ${ }^{7}$

El diagnóstico se hace por medio de una ecosonografía donde se aprecia una hepatomegalia total con múltiples imágenes redondeadas, hipoecoicas y altamente vascularizadas. En cuanto al tratamiento, existe alto sustento científico que demuestra la adherencia y respuesta del propanolol. ${ }^{8}$ En este trabajo se puede observar un caso clínico de un infante de dos meses que comienza con distensión abdominal en el cuadrante abdominal superior derecho acompañado de ictericia, adinamia, entre otras; por lo que el diagnóstico se basó principalmente en la 
clínica junto con la biometría hemática la cual demostró una trombocitopenia que está en relación a un secuestro plaquetario por los tumores vasculares, ecosonografía y TMC.

Actualmente el hemangioma hepático es habitual encontrarlo en la práctica clínica, se puede detectar bajo la utilización de varias exploraciones como la ecografía abdominal, tomografía computarizada multiforme (TCM), además de pruebas de laboratorio. En cuanto a complicaciones es benigno y existe muy poco sustento científico que certifique complicaciones futuras en infantes que lo han padecido, esto es lo que hace novedoso a este caso pues aquí se producen estas complicaciones que son muy raras. ${ }^{8}$

Es sumamente raro encontrar un hemangioendotelioma hepático de lactante que se complique con insuficiencia cardiaca, como es este caso y peor aún que no se encuentre shunts arteriovenosos en la angiografía. Una fortaleza del presente caso es poder conocer a detalle el reporte y la evolución del infante desde su ingreso hasta su egreso.

\section{Conclusiones}

El diagnóstico de hemangioendotelioma hepático del lactante es una patología rara y más aún que su forma de presentación tenga complicaciones graves y el paciente fallezca. Es importante resaltar que el diagnóstico se puede hacer sin la exigencia de una confirmación histológica cuando los hallazgos clínicos, analíticos y de imagen son muy sugestivos, sobre todo en los pacientes con menos de seis meses de edad.

\section{Conflicto de intereses}

Ninguno declarado por los autores.

\section{Financiación}

Autofinanciado.

\section{Agradecimientos}

Ninguno declarado por los autores.

\section{Referencias}

1. Figuera Z, Delgado M, López C, Morao C, León P, Reymúndez M et al. Hemangiomatosis hepática en lactantes: diagnóstico y evolución. hospital j. m. de los ríos. caracas - venezuela. Gen [en línea]. 2011 [citado 27 de abril de 2017]; 65(1): 38-41. Disponible en: http://www.scielo.org.ve/scielo.php?script=sci_arttext\&pid=S0016-35032011000100009\&lng=es

2. Hemangioendotelioma [en línea]. Medciclopedia. 2017. Recuperado a partir de: http://www.iqb.es/cancer/hemangioendotelioma/hemangioendotelioma.htm

3. Rosales Labrada Regis Gerardo, Rosales Pupo Lilian Beatriz, Almira Gonzalez Ernesto Fidel, Almira Gómez Carlos Rafael, Domínguez Morales Jesús Ignacio. Presentación de un niño con hemangioendotelioma hepático. ccm [en línea]. 2017 [citado 16 de mayo de 2017];21(1): 286-294. Disponible en: http://scielo.sld.cu/scielo.php?script=sci arttext\&pid=S1560-43812017000100025\&lng=es

4. Mclaughlin M, O'connor N, Newborn Skin: Part II. Birthmarks. afp [en línea]. 2008 [16 de mayo de 2017];77(1):56-60. Disponible en: https://www.aafp.org/afp/2008/0101/p56.html

5. Yagima García, Leidelén Sosa, Yurisandra Jiménez. Hemangioendotelioma hepático infantil: dos desenlaces diferentes. VC [en línea]. 2016 [citado 25 de mayo de 2017]; 20 (1): 74-80. Disponible en: http://scielo.sld.cu/pdf/mdc/v20n1/mdc12116.pdf

6. Araújo A, Maciel I, Costa J, Vieira A, Enes C. Hemangioendotelioma hepático infantil. Un caso multifocal y bilobular, asintomático, con regresión espontánea. ap [en línea]. 2008. [citado 25 de mayo de 2017];68(5): 507-510. Disponible en: http://analesdepediatria.elsevier.es/es/pdf/S1695403308701905/S300/

7. Leal N, Santamaría M, Gámez M, Murcia J, López Gutiérrez et al. Hemangioendotelioma multifocal hepático infantil ¿Es siempre un tumor benigno? cp [en línea]. 2004 [citado 25 de mayo de 2017];17: 8-11. Disponible en: https://www.secipe.org/coldata/upload/revista/2004;17.8-11.pdf

8. Palacios J, Carrasquel V, Jaramillo E, Herrera D. Hemangioma hepático infantil tratado con propanolol. RM [en línea]. 2014. [citado 25 de mayo de 2017];18: 130-139. Disponible en: http://www.medigraphic.com/pdfs/revmexcirped/mcp-2014/mcp143d.pdf 